\title{
Extension of the Buchalla-Safir bound
}

\author{
L. Lavoura* \\ Universidade Técnica de Lisboa \\ Centro de Física das Interacções Fundamentais \\ Instituto Superior Técnico, 1049-001 Lisboa, Portugal
}

18 February 2004

\begin{abstract}
I provide a simple derivation of the Buchalla-Safir bound on $\gamma$. I generalize it to the case where an upper bound on the phase of the penguin pollution is assumed. I apply the Buchalla-Safir bound, and its generalization, to the recent Belle data on $C P$ violation in $B \rightarrow \pi^{+} \pi^{-}$.
\end{abstract}

\section{Introduction}

$C P$ violation in $B_{d}^{0}-\bar{B}_{d}^{0}$ mixing and in the decays of those mesons to $\pi^{+} \pi^{-}$is parametrized by

$$
\lambda=\frac{q}{p} \frac{\bar{A}}{A}
$$

where $q / p$ relates to $B_{d}^{0}-\bar{B}_{d}^{0}$ mixing, $A$ is the amplitude for $B_{d}^{0} \rightarrow \pi^{+} \pi^{-}$, and $\bar{A}$ is the amplitude for $\bar{B}_{d}^{0} \rightarrow \pi^{+} \pi^{-}$[1]. Two $C P$-violating quantities can be measured:

$$
\begin{aligned}
S & =\frac{2 \operatorname{Im} \lambda}{1+|\lambda|^{2}}, \\
C & =\frac{1-|\lambda|^{2}}{1+|\lambda|^{2}} .
\end{aligned}
$$

Let

$$
\frac{q}{p}=\exp (-2 i \tilde{\beta})
$$

In the Standard Model (SM), $\tilde{\beta}=\beta$ and the sine of $2 \beta$ is measured [2] through $C P$ violation in $B_{d}^{0} / \bar{B}_{d}^{0} \rightarrow \psi K_{S}$ :

$$
\sin 2 \beta=0.736 \pm 0.049 .
$$

In the SM $\beta$ must be smaller than $\pi / 4$, hence $\cos 2 \beta$ is assumed positive.

\footnotetext{
*E-mail: balio@cfif.ist.utl.pt
} 
Together with eq. (4), I shall assume that, as in the SM,

$$
\frac{\bar{A}}{A}=\frac{e^{-i \gamma}+z}{e^{i \gamma}+z},
$$

where $\gamma$ is another $C P$-violating phase, which we would like to measure too. In the $\mathrm{SM}, 0 \leq \gamma \leq \pi-\beta$. The parameter $z$ represents the 'penguin pollution', an annoying contribution from penguin diagrams which we must somehow circumvent if we want to get at $\gamma$.

Buchalla and Safir (BS) [3] have found a solution to the following problem. Suppose that

- one has measured $\sin 2 \tilde{\beta}$ and $S$,

- one has found that $S>-\sin 2 \tilde{\beta}$,

- one assumes the validity of the SM, and

- one assumes that $\operatorname{Re} z>0$.

Is it then possible to find a lower bound on $\gamma$ stronger than $\gamma \geq 0$ ? The solution to this problem, as given by BS, is

$$
\gamma>\frac{\pi}{2}-\arctan \frac{S-\tau+\tau \sqrt{1-S^{2}}}{\tau S+1-\sqrt{1-S^{2}}},
$$

where

$$
\tau \equiv \frac{\sin 2 \tilde{\beta}}{1-\sqrt{1-\sin ^{2} 2 \tilde{\beta}}}
$$

and the square roots in eqs. (77) and (8) are, by definition, positive.

In this Letter I provide a simple derivation of the BS bound, which does not rely on any assumptions about the quark mixing matrix. I also consider the realistic situation where both $S$ and $C$ have been measured; this allows one to put a stronger bound on $\gamma$ than when one knows only $S$. Inspired by the result, quoted by BS, of a computation of $z$ yielding

$$
\arg z=0.15 \pm 0.25
$$

I furthermore consider the situation where one assumes an upper bound on $|\arg z|$. Finally, I apply the BS bound, and its extensions, to the most recent measurements of $S$ and $C$ made public by the experimental collaboration Belle [4].

\section{The Buchalla-Safir bound}

I define

$$
\begin{aligned}
x & \equiv \lambda \exp (2 i \tilde{\beta}) \\
& =\frac{e^{-i \gamma}+z}{e^{i \gamma}+z} .
\end{aligned}
$$


Then,

$$
C=\frac{1-|x|^{2}}{1+|x|^{2}},
$$

and I furthermore define

$$
\begin{aligned}
I & \equiv \frac{2 \operatorname{Im} x}{1+|x|^{2}} \\
F & \equiv \frac{|1-x|^{2}}{1+|x|^{2}} \\
& =1-\frac{2 \operatorname{Re} x}{1+|x|^{2}}
\end{aligned}
$$

Clearly,

$$
0 \leq F \leq 2
$$

and

$$
C^{2}+I^{2}+F^{2}=2 F
$$

Solving eq. (10) for $z$, one finds

$$
z=-\cos \gamma+\frac{-I+i C}{F} \sin \gamma
$$

Equation (16) has an indeterminacy at the singular point $C=I=F=0 \Leftrightarrow x=1$, i.e. when $\sin \gamma=0$, for arbitrary $z$.

From eq. (16) it follows in particular that

$$
F(\cos \gamma+\operatorname{Re} z)+I \sin \gamma=0 .
$$

Equation (17) has been first written down by Botella and Silva [5]. It leads to the bound

$$
|\operatorname{Re} z| \leq \frac{\sqrt{F^{2}+I^{2}}}{F}
$$

where $\sqrt{F^{2}+I^{2}}$ is positive by definition. The solution to eq. (17) may be written in the form

$$
\gamma=\xi+\chi
$$

where (by definition)

- $\xi$ is independent of $\operatorname{Re} z$, and

- $\chi=0$ or $\chi=\pi$ when $\operatorname{Re} z=0$.

One finds

$$
\begin{aligned}
\cos \xi & =\frac{-I}{\sqrt{F^{2}+I^{2}}} \\
\sin \xi & =\frac{F}{\sqrt{F^{2}+I^{2}}}
\end{aligned}
$$


and

$$
\sin \chi=\frac{F \operatorname{Re} z}{\sqrt{F^{2}+I^{2}}}
$$

While $\xi$ is perfectly defined by eqs. (20) and (21), $\chi$ as given by eq. (22) suffers from the twofold ambiguity

$$
\chi \rightarrow \pi-\chi
$$

Assuming, as Buchalla and Safir have done, that Re $z>0$, we see from eqs. (21) and (22) that both $\xi$ and $\chi$ are angles either of the first or of the second quadrant. The BuchallaSafir condition $\operatorname{Re} z>0$ implies the lower bound on $\gamma$

$$
\begin{aligned}
\gamma & >\xi \\
& =\arccos \frac{-I}{\sqrt{F^{2}+I^{2}}}
\end{aligned}
$$

together with $\gamma<\xi+\pi$ too. Notice that

$$
d \xi=\frac{F d I-I d F}{F^{2}+I^{2}}
$$

Equation (24) does provide a lower bound on $\gamma$ but, unfortunately, one has to deal with discrete ambiguities. These occur because we are able to measure $C$ but unable to measure $I$ and $F$; rather, we only know $\sin 2 \tilde{\beta}$ and $S$. Now,

$$
\begin{aligned}
I & =\frac{2 \operatorname{Re} \lambda}{1+|\lambda|^{2}} \sin 2 \tilde{\beta}+S \cos 2 \tilde{\beta} \\
F & =1-\frac{2 \operatorname{Re} \lambda}{1+|\lambda|^{2}} \cos 2 \tilde{\beta}+S \sin 2 \tilde{\beta}
\end{aligned}
$$

Assuming that $\sin 2 \tilde{\beta}, S$, and $C$ are known, there is a fourfold ambiguity in $I$ and $F$, since the signs of

$$
\begin{aligned}
\frac{2 \operatorname{Re} \lambda}{1+|\lambda|^{2}} & =\sqrt{1-C^{2}-S^{2}}, \\
\cos 2 \tilde{\beta} & =\sqrt{1-\sin ^{2} 2 \tilde{\beta}}
\end{aligned}
$$

remain unknown. Using eqs. (25)-(29),

$$
\frac{d \xi}{d C^{2}}=\frac{-S-\sin 2 \tilde{\beta}}{2\left(F^{2}+I^{2}\right) \sqrt{1-C^{2}-S^{2}}} .
$$

(Remember that the sign of $\sqrt{1-C^{2}-S^{2}}$ is, for the moment, arbitrary.)

Thus, given $C, S$, and $\sin 2 \tilde{\beta}$, there are in reality four different angles $\xi$ :

- $\xi_{1}$, in which both $\sqrt{1-C^{2}-S^{2}}$ and $\cos 2 \tilde{\beta}$ are positive,

- $\xi_{2}$, in which $\cos 2 \tilde{\beta}$ is positive but $\sqrt{1-C^{2}-S^{2}}$ is negative, 
- $\xi_{3}$, in which both $\sqrt{1-C^{2}-S^{2}}$ and $\cos 2 \tilde{\beta}$ are negative, and

- $\xi_{4}$, in which $\sqrt{1-C^{2}-S^{2}}$ is positive but $\cos 2 \tilde{\beta}$ is negative.

Since $F$ remains invariant, and $I$ changes sign, when $\sqrt{1-C^{2}-S^{2}}$ and $\cos 2 \tilde{\beta}$ change sign simultaneously, we find that $\xi_{3}=\pi-\xi_{1}$ and $\xi_{4}=\pi-\xi_{2}$. From the assumption that $\operatorname{Re} z>0$, and taking into account the indeterminacy in the signs of $\sqrt{1-C^{2}-S^{2}}$ and $\cos 2 \tilde{\beta}$, one can only deduce that $\gamma$ must lie in between $\xi_{k}$ and $\xi_{k}+\pi$ for all $k=1,2,3$, and 4 .

Let us now assume, with BS, the validity of the SM. Then $\cos 2 \tilde{\beta}$ is positive and only the values $\xi_{1}$ and $\xi_{2}$ are allowed for $\xi$. This produces the lower bound

$$
\gamma>\min \left(\xi_{1}, \xi_{2}\right) \text {. }
$$

This lower bound is valid in the SM when $C, S$, and $\sin 2 \tilde{\beta}$ are known. It still depends on $C^{2}$, since $\xi_{1}$ and $\xi_{2}$ contain $\sqrt{1-C^{2}-S^{2}}$. Consideration of eq. (30), however, shows that, when $S>-\sin 2 \tilde{\beta}, \xi_{1}$ decreases and $\xi_{2}$ increases with increasing $C^{2}$. Moreover, at the maximum allowed value of $C^{2}$, i.e. when $C^{2}=1-S^{2}$, one has $\xi_{1}=\xi_{2}$, since in general $\xi_{1}$ and $\xi_{2}$ only differ through the sign of $\sqrt{1-C^{2}-S^{2}}$, and that square root becomes zero when $C^{2}=1-S^{2}$. This immeadiately leads to the BS bound: if $S>-\sin 2 \tilde{\beta}$, then $\gamma>\xi_{2}\left(C^{2}=0\right)$. It can be shown [5] that, though different in appearance, this bound coincides with the one in eq. (77).

One thus concludes that, if one assumes that $\cos 2 \tilde{\beta}>0$, then

$$
\left\{\begin{array}{l}
\gamma>\xi_{2}\left(C^{2}=0\right) \Leftarrow S>-\sin 2 \tilde{\beta} \\
\gamma>\xi_{1}\left(C^{2}=0\right) \Leftarrow S<-\sin 2 \tilde{\beta} .
\end{array}\right.
$$

This may be put in a more transparent way if one defines

$$
\begin{aligned}
\varphi & \equiv \frac{1}{2} \arcsin S, \\
\alpha & \equiv \pi-\tilde{\beta}-\gamma .
\end{aligned}
$$

The lower bound on $\gamma$ may then be rewritten as an upper bound on $\alpha$ :

$$
\left\{\begin{aligned}
\alpha<\frac{\pi}{2}-\varphi & \Leftarrow \varphi>-\tilde{\beta}, \\
\alpha<\pi+\varphi & \Leftarrow \varphi<-\tilde{\beta} .
\end{aligned}\right.
$$

The discontinuity of the bound at $\varphi=-\tilde{\beta}$ should not come as a surprise. The point $C=0, S=-\sin 2 \tilde{\beta}$ allows the singularity $C=I=F=0$ referred to earlier. When $C=I=F=0, \gamma$ may be either 0 or $\pi$, independently of any assumption on $z$. Therefore no lower bound on $\gamma$ may be derived if the experimentally allowed region for $C$ and $S$ includes that point.

It should be stressed that this derivation of the Buchalla-Safir bound on $\gamma$, or on $\alpha$, contains basically no physical assumptions. Only eqs. (11)-(4) and (6), together with $\cos 2 \tilde{\beta}>0$ and $\operatorname{Re} z>0$, are assumed. No assumptions are needed about the physics contained in the decay amplitudes, about the quark mixing matrix, or, indeed, about 
anything else; the sole crucial assumption is $\operatorname{Re} z>0$. The Buchalla-Safir bound is purely algebraic.

I now return to the general case where one does not assume the SM. Then, $\gamma$ may be either positive or negative and, from the assumption that $\operatorname{Re} z>0$, it is only possible to produce a lower bound on $|\gamma|$, never on $\gamma$ itself. Indeed, given the fourfold ambiguity in the determination of $F$ and $I$, and the twofold ambiguity in the determination of $\chi$ - see eq. (23) - there are eight solutions to eq. (17) for $\gamma$. Since, when $\sqrt{1-C^{2}-S^{2}}$ and $\cos 2 \tilde{\beta}$ change sign simultaneously, $I$ changes sign while $F$ does not change, it is obvious from eq. (17) that those eight solutions pair in four sets through the transformation $\gamma \rightarrow-\gamma$. Therefore, only a bound on $|\gamma|$ is possible. Now, computing

$$
\tan ^{2} \xi_{1}\left(C^{2}=0\right)-\tan ^{2} \xi_{2}\left(C^{2}=0\right)=\frac{-4 \sqrt{1-S^{2}} \sqrt{1-\sin ^{2} 2 \tilde{\beta}}}{(\sin 2 \tilde{\beta}-S)^{2}},
$$

where the square roots in the right-hand side are positive by definition, one finds that $\left|\tan \xi_{1}\left(C^{2}=0\right)\right|$ is always smaller than $\left|\tan \xi_{2}\left(C^{2}=0\right)\right|$. Hence,

$$
|\gamma|>\arctan \left|\tan \xi_{1}\left(C^{2}=0\right)\right| \text {. }
$$

Using again $\varphi$ as defined in eq. (33), one concludes that

$$
|\gamma|>|\tilde{\beta}+\varphi|
$$

which is valid in any model provided Re $z>0$ - and provided the basic equations (11) -(4) and (6) hold, of course.

\section{Assuming an upper limit on $|\arg z|$}

In their work [3], Buchalla and Safir have quoted the result of a computation (in the context of the Standard Model) of $z$ as yielding the result in eq. (9). They have thereby justified their assumption $\operatorname{Re} z>0$. In this section I shall consider a different assumption,

$$
|\cot \arg z|>L
$$

where $L$ is some positive number. Clearly, this assumption is complementary to $\operatorname{Re} z>0$; while $\operatorname{Re} z>0$, by itself alone, leaves $\cot \arg z$ completely arbitrary, eq. (39), by itself alone, does not provide any information on whether $\operatorname{Re} z$ is positive or negative. If $L$ is, for instance, taken equal to 1 , then eq. (39) is well justified by eq. (9).

In order to find the consequences of the assumption in eq. (39), I return to eq. (16) and therefrom derive that

$$
C \cot \arg z+F \cot \gamma+I=0
$$

Hence,

$$
|\cot \arg z|>L \Leftrightarrow \cot \gamma<\frac{-I-L|C|}{F} \quad \text { or } \quad \cot \gamma>\frac{-I+L|C|}{F} .
$$


Clearly, this condition makes smaller the range for $\gamma$ allowed by $\operatorname{Re} z>0$ alone; that range, remember, is given by $\xi<\gamma<\xi+\pi$, where $\xi$ belongs either to the first or to the second quadrant and $\cot \xi=-I / F$.

Let us now assume the validity of the SM. Then $\gamma \leq \pi-\beta$ and the relevant bound on $\gamma$ following from eq. (39) is the lower bound

$$
\begin{aligned}
\cot \gamma & <\frac{-I-L|C|}{F} \\
& =\frac{-\sqrt{1-C^{2}-S^{2}} \sin 2 \tilde{\beta}-S \cos 2 \tilde{\beta}-L|C|}{1-\sqrt{1-C^{2}-S^{2}} \cos 2 \tilde{\beta}+S \sin 2 \tilde{\beta}} .
\end{aligned}
$$

This bound depends on the measured values of $C, S, \sin 2 \tilde{\beta}$ and, besides, since $\cos 2 \tilde{\beta}$ is positive in the SM, it depends on the sign of $\sqrt{1-C^{2}-S^{2}}$.

\section{Application to the Belle results}

The BS bound applies to the situation where $S$ has been measured while $C$ remains unknown but, in reality, both the BABAR and Belle Collaborations are able to measure $S$ and $C$ simultaneously and with comparable accuracy. Early results made public by BABAR [6] are

$$
\begin{aligned}
& S \in[-0.54,0.58], \\
& C \in[-0.72,0.12]
\end{aligned}
$$

at $90 \%$ Confidence Level (C.L.). In this section I shall rather use the latest results by the Belle Collaboration [4]. Belle measures $S$ and $C$ to be both negative and not satisfying the constraint $S^{2}+C^{2} \leq 1$; enforcing the latest constraint, the Belle Collaboration has presented the allowed regions for $C$ and $S$ displayed in fig. 1. The point $C=0, S=$ $-\sin 2 \beta$ is disallowed at 99.9157 C.L., and therefore setting a BS lower bound on $\gamma$ is possible. Assuming the SM, the lower bound on $\gamma$ that I shall consider is given by the inequality (42), where $\sqrt{1-C^{2}-S^{2}}$ may be either positive or negative - we must use, for each pair of values for $S$ and $C$, the sign of $\sqrt{1-C^{2}-S^{2}}$ yielding the less stringent bound. I shall assume fixed values for $\sin 2 \tilde{\beta}=0.736$ and $\cos 2 \tilde{\beta}=+\sqrt{1-0.736^{2}}$. For $L$ I shall take the four values $L=0$ - the case relevant for the BS bound, where $\operatorname{Re} z>0$, but no lower bound on $|\cot \arg z|$, is assumed-and $L=\cot 0.9$, cot 0.65 , and $\cot 0.4$, corresponding to the $3 \sigma, 2 \sigma$, and $1 \sigma$ bounds, respectively, following from eq. (9).

I performed scans of the allowed regions in the $(C, S)$ plane advocated by the Belle Collaboration. For each value of the pair $(C, S)$, and for each value of $L$, I computed the corresponding lower bound on $\gamma$. The results are the following. If one takes the $68.3 \%$ C.L. domain of Belle, then $\gamma>21.8^{\circ}$ if $L=0, \gamma>42.3^{\circ}$ if $L=\cot 0.9, \gamma>58.3^{\circ}$ if $L=\cot 0.65$, and $\gamma>93.6^{\circ}$ if $L=\cot 0.4$. When one uses the the region allowed by Belle at $95.45 \%$ C.L., one obtains $\gamma>12.3^{\circ}$ if $L=0, \gamma>24.1^{\circ}$ if $L=\cot 0.9, \gamma>33.9^{\circ}$ if $L=\cot 0.65$, and $\gamma>53.7^{\circ}$ if $L=\cot 0.4$. Considering at last the $99.73 \%$ C.L. limits of Belle, one gets $\gamma>3.6^{\circ}$ if $L=0, \gamma>6.6^{\circ}$ if $L=\cot 0.9, \gamma>8.9^{\circ}$ if $L=\cot 0.65$, and $\gamma>12.5^{\circ}$ if $L=\cot 0.4$; these very loose bounds reflect the proximity to this region of the point $C=0, S=-\sin 2 \beta$, for which no lower bound on $\gamma$ is possible any more. 


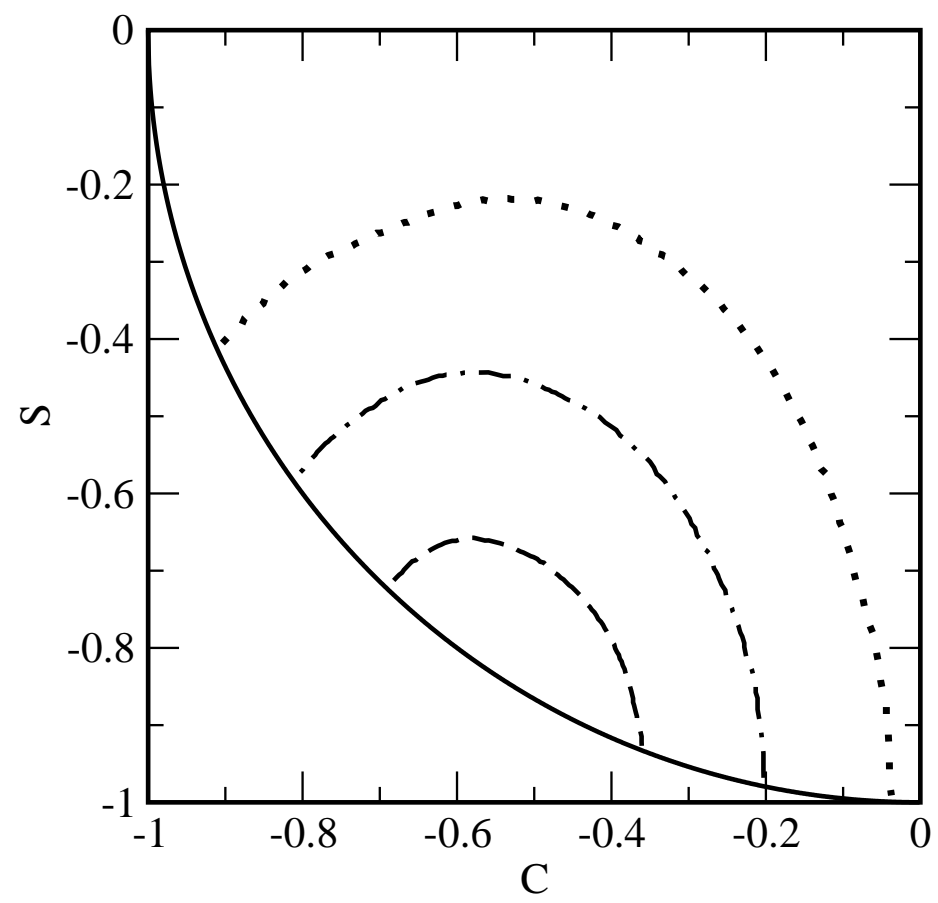

Figure 1: The latest results of the Belle Collaboration for $S$ and $C$. The full line bounds the circle defined by the condition $C^{2}+S^{2} \leq 1$. Within that circle, the dashed line bounds the region allowed by Belle at $68.3 \%$ C.L., the dot-dashed line bounds the region allowed at $95.45 \%$ C.L., and the dotted line bounds the region allowed at $99.73 \%$ C.L.

It is evident from the results above that assuming $|\cot \arg z|>L$, with a non-zero $L$, may greatly improve the lower bound on $\gamma$ that one obtains from the BS condition $\operatorname{Re} z>0$ alone.

\section{Conclusions}

I have shown in this Letter that the Buchalla-Safir lower bound on $\gamma$ is a purely algebraic consequence of the assumption $\operatorname{Re} z>0$; the latter assumption follows from a computation of $z$ within the Standard Model but, after that computation, the derivation of the BS bound itself requires no physics. I have emphasized that a better lower bound on $\gamma$ may be obtained if one considers that, besides $S$, also $C$ is known. I have improved the BS bound by assuming, above and beyond $\operatorname{Re} z>0$, a lower bound on $|\cot \arg z|$. I have emphasized the fact that the presence, within the experimentally allowed region, of the point $(S, C)=(-\sin 2 \beta, 0)$, prevents one from putting a lower bound on $\gamma$. I have applied the derived bounds to the $(S, C)$ domains allowed by the most recent results made public by the Belle Collaboration.

Acknowledgements I am grateful to João Paulo Silva for introducing the BS bound to me, and for discussions; his paper with Botella [5] motivated and strongly influenced the 
present Letter. I am also grateful to Jorge Crispim Romão, who has helped me willingly

for several times in software matters. This work has been supported by the Portuguese Fundação para a Ciência e a Tecnologia under the project CFIF-Plurianual.

\section{References}

[1] For an introduction to $C P$ violation in the $B$ system see G. C. Branco, L. Lavoura, and J. P. Silva, CP violation (Oxford, England: Oxford University Press, 1999).

[2] T. E. Browder, "Results on the CKM angle $\phi_{1}(\beta)$," hep-ex/0312024, to appear in the Proceedings of the 2003 Lepton-Photon Conference.

[3] G. Buchalla and A. S. Safir, "Model-independent bound on the unitarity triangle from $C P$ violation in $B \rightarrow \pi^{+} \pi^{-}$and $B \rightarrow \psi K_{S}$," hep-ph/0310218.

[4] K. Abe et al. (The Belle Collaboration), "Observation of large $C P$ violation and evidence for direct $C P$ violation in $B^{0} \rightarrow \pi^{+} \pi^{-}$decays," hep-ex/0401029.

[5] F. J. Botella and J. P. Silva, "Bounds on $\gamma$ from $C P$-violation measurements in $B \rightarrow \pi^{+} \pi^{-}$and $B \rightarrow \psi K_{S}, "$ hep-ph/0312337.

[6] B. Aubert et al. (BABAR Collaboration), "Measurements of branching fractions and $C P$-violating asymmetries in $B^{0} \rightarrow \pi^{+} \pi^{-}, K^{+} \pi^{-}, K^{+} K^{-}$decays," Physical Review Letters 89, 281802 (2002). 